\title{
Bilateral Osteochondritis Dissecans of the Femoral Condyles in Both Knees: A Report of Two Sibling Cases
}

Jae-Hoon Jeong, $\mathrm{MD}^{1}$, Randy Mascarenhas, $\mathrm{MD}^{2}$, and Hang Seob Yoon, $\mathrm{MD}^{1}$

${ }^{1}$ Department of Orthropaedic Surgery, Bone and Joint Institute, Seoul Wooridul Hospital, Seoul, Korea; ${ }^{2}$ Section of Orthopaedic Surgery, University of Manitoba School of Medicine, Winnipeg, MB, Cananda

Osteochondritis dissecans (OCD) of both femoral condyles is very rare, with no previously reported cases of bilateral OCD of both knees in two siblings. We report on a brother and sister with both femoral condyle OCD with a description of surgical technique and clinical results. Fixation using headless compressive screws, osteochondral autologous transplantation and autologous chondrocyte implantation were all successful.

Keywords: Osteochondritis dissecans, Bilateral, Both femoral condyle, Knee, Autologous chondrocyte implantation, Open osteochondral autograft

Various causes of osteochondritis dissecans (OCD) have been proposed including trauma and constitutional factors, but consensus on the etiology of OCD remains elusive. We present two cases of bilateral OCD of femoral condyles of both knees in a 32-year-old female and her 29-year-old younger brother. Neither had a history of trauma to their knees. The female patient was treated with osteochondral autograft transplantation (OAT) and autologous chondrocyte implantation (ACI), while arthroscopic fixation of a displaced osteochondral fragment with headless compressive screws (Acutrak; Acumed, Hillsboro, OR, USA) was performed in the male patient.

Received January 24, 2013; Revised (1st) March 29, 2013;

(2nd) April 15, 2013; Accepted April 18, 2013

Correspondence to: Jae-Hoon Jeong, MD

Department of Orthropaedic Surgery, Bone and Joint Institute, Seoul Wooridul Hospital, 70 Haneul-gil, Gangseo-gu, Seoul 157-823, Korea Tel: +82-2-2660-7500, Fax: +82-2-2660-7599

E-mail: arthrokorea@hotmail.com

This is an Open Access article distributed under the terms of the Creative Commons Attribution Non-Commercial License (http://creativecommons.org/licenses/by-nc/3.0/) which permits unrestricted non-commercial use, distribution, and reproduction in any medium, provided the original work is properly cited.

\section{Case Reports}

\section{Case 1}

A 32-year-old female visited our hospital with a complaint of right knee pain. She had come three months ago for similar symptoms and had been diagnosed with OCD of the right knee based on clinical presentation and imaging studies obtained at that time. At the latest presentation, the pain increased in severity and the patient required a cane for ambulation due to a catching sensation in her right knee. Physical examination revealed moderate swelling and stiffness of the right knee. Localized tenderness was noted on the lateral and medial joint lines and twenty degrees of passive extension was lost.

Standing weight-bearing posterior-anterior radiographs were obtained and revealed irregularities of bilateral femoral condyles in both knees on the weight-bearing portion of the femur (Fig. 1). Magnetic resonance imaging (MRI) showed a fragment in the center of the both femoral condyles with bone marrow edema. A fissure between the articular fragment and femoral bone base was also seen. Additionally, a $2.8 \mathrm{~cm}$ by $2.5 \mathrm{~cm}$ displaced articular loose body shadow was noticed on the lateral femoral condyle.

\section{1) Operative technique}

The patient was brought to the operating room and prepped and draped in the standard sterile fashion. Arthroscopic examination was performed first and findings revealed a $2.8 \mathrm{~cm}$ 

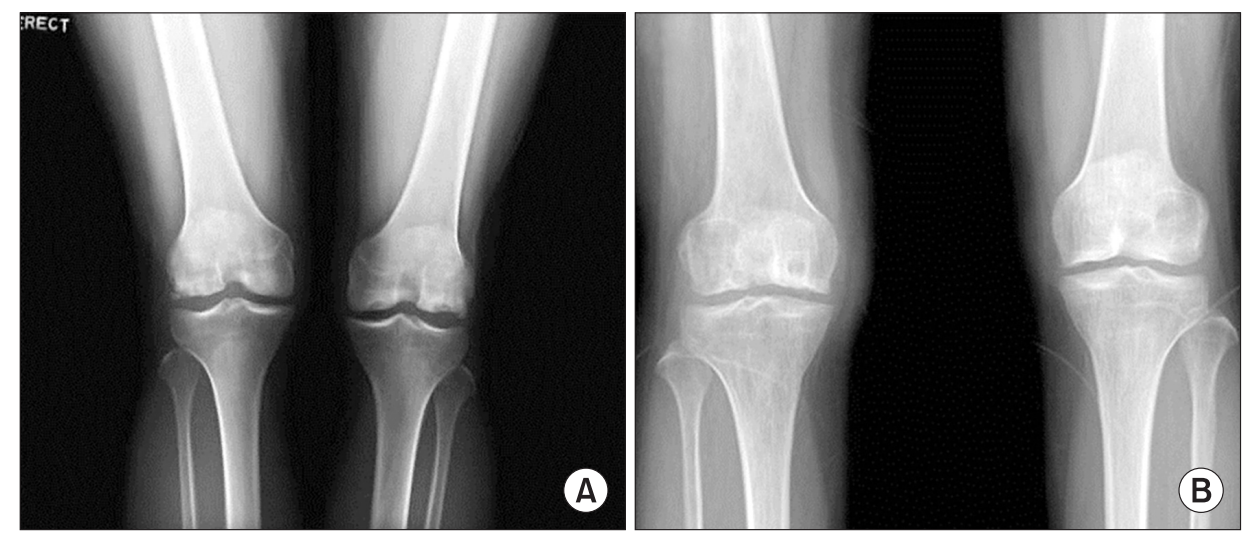

Fig. 1. Anteroposterior radiographs of the knee show that both femoral condyles have large bony irregularities with shallow, similar sized excavations bilaterally in both patients. (A) A 31-year-old female patient (patient 1). (B) A 28-year-old male patient (patient 2).

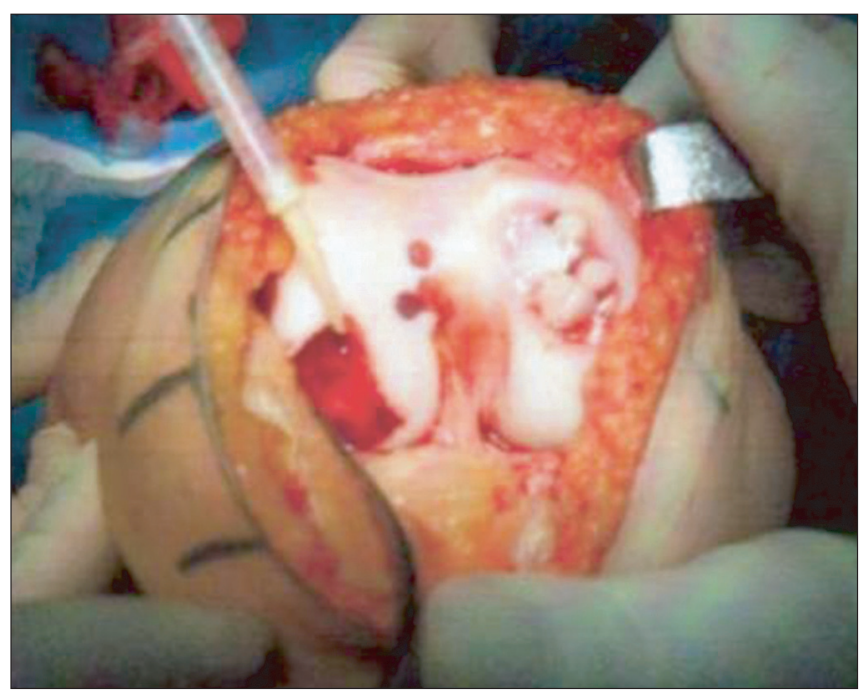

Fig. 2. Autologous chondrocyte implantation of the lateral femoral condyle in the right knee of patient 1 with osteochondral autologous transplantation on the medial side.

by $2.5 \mathrm{~cm}$ displaced osteochondral fragment from the lateral femoral condyle. The OCD defect was $0.5 \mathrm{~cm}$ in depth and contained a subchondral cyst and degeneration. A similar sized loose osteochondral lesion was also found on the medial femoral condyle with degenerative subchondral bone. It was decided that there would be a lower potential for healing if the fragments were simply re-attached. A decision was thus made to proceed with ACI for the lateral lesion and OAT for the medial lesion. Chondrocytes were harvested and the patient was brought back for subsequent operation five weeks later. An 8-cm midline skin incision was made on the right knee and an arthrotomy was performed. The lateral femoral condyle defect was treated with ACI (Fig. 2). Four osteochondral grafts were obtained from the superior aspect of the femoral condyle and trochlea using a special chisel (OATS; Arthrex, Naples, FL, USA). After implanting the donor cylinders using a press fit technique on the medial femoral condyle, the free space between the grafts was filled with remnant chondrocytes from the ACI. The patient was not allowed weight-bearing for six weeks and passive and active range of motion exercises were performed during this period. The patient was followed up for two years after surgical treatment. Her Lysholm score and visual analogue scale score were improved from 32 preoperatively to 90 at the last follow-up and from 9 preoperatively to 1 at the last follow-up, respectively.

\section{Case 2}

The 28-year-old brother of the aforementioned patient presented six months after her surgery with painful swelling of his right knee lasting for one day. He routinely experienced sharp pain with weight-bearing despite the lack of a traumatic injury to the right knee. Physical examination revealed an antalgic gait requiring the use of crutches for ambulation. Locking was experienced as the knee was flexed and tenderness was localized in the medial joint line. Pain-free range of motion was limited to 20-50 degrees. Standard radiographs (Fig. 1B) and MRI (Fig. 3) showed findings similar to those of the patient described in case one.

\section{1) Operative technique}

Arthroscopic examination of the right knee revealed a $2 \mathrm{~cm}$ by $3 \mathrm{~cm}$ round fragment from the medial femoral condyle. The peripheral margin was clean and the edge of the posterolateral portion of the medial femoral condyle was partially attached to the fragment. Before re-attaching the fragment, a subchondral crater was curetted on the medial femoral condyle to remove fibrous tissue. The lesion was also drilled to improve vascularity on the femoral side.

Next, with arthroscopic visualization through the superolateral portal, reduction of the fragment was performed and a Kirschner 

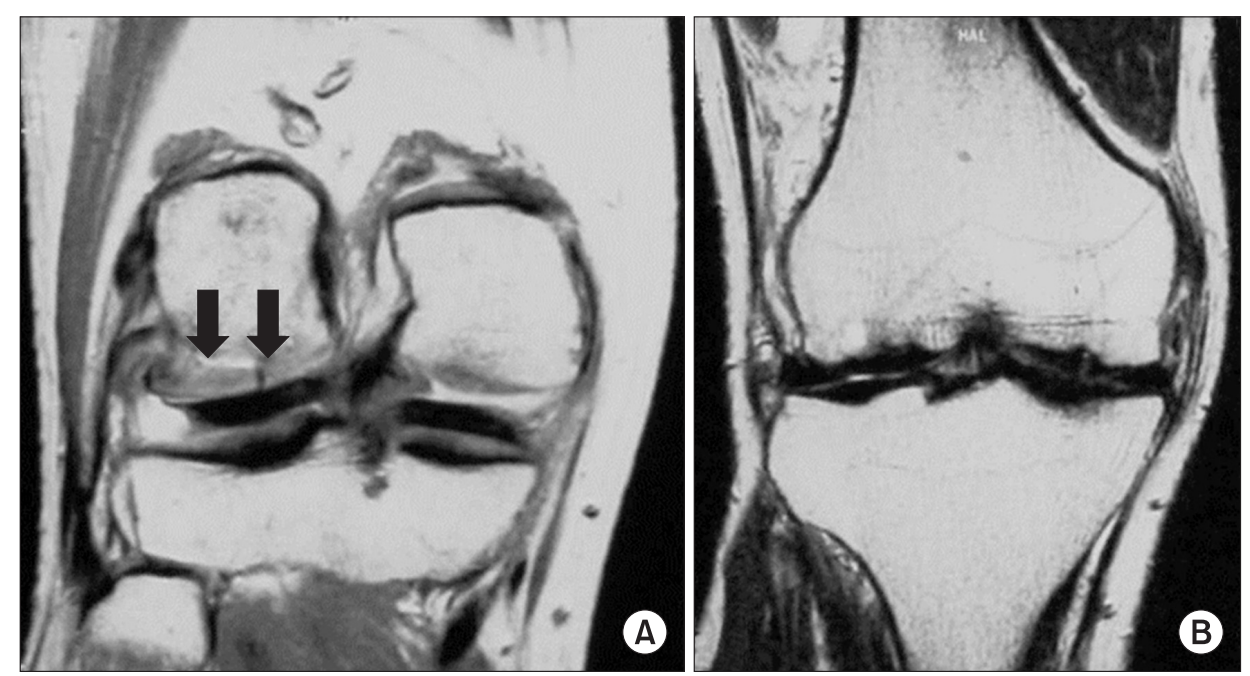

Fig. 3. (A) An magnetic resonance imaging of the right knee in patient 2 showed signal changes in both femoral condyles and a large bone-like density in the posterolateral compartment. Bony edema and low signal was also noted between the fragment and its femoral origin. (B) The lesions are identified down to the subchondral bone of both femoral condyles.
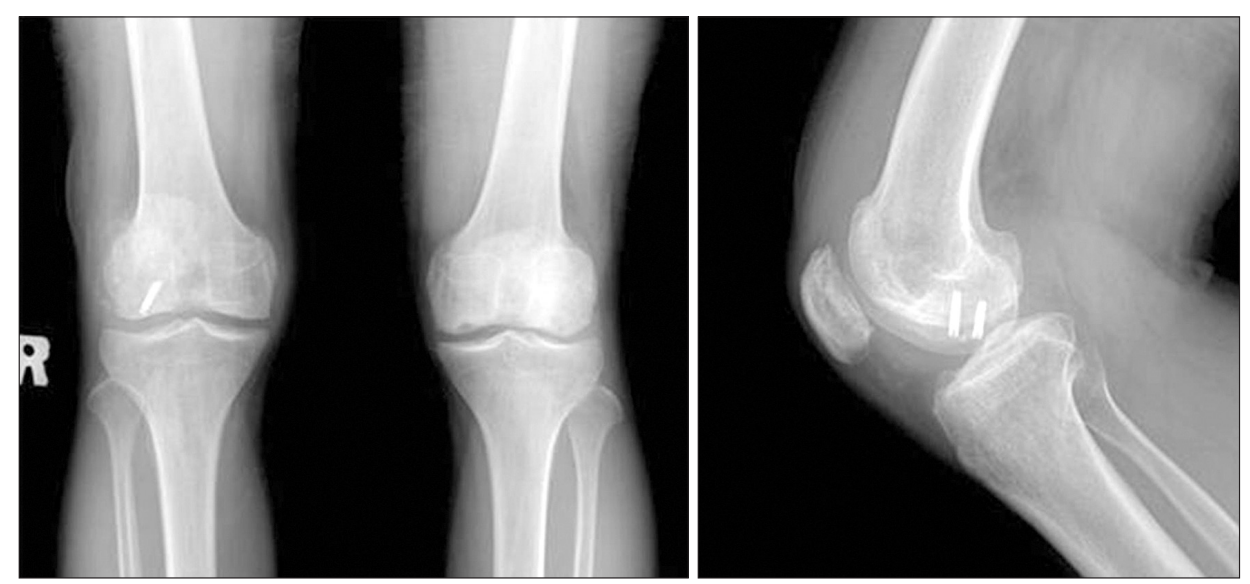

Fig. 4. Postoperative radiographs of the right knee in patient 2 after arthroscopic fixation of a loose articular fragment with Acutrak screws.

wire was temporarily inserted. Two headless compression screws (Acutrak) were inserted in 120 degrees of knee flexion using the medial portal (Fig. 4). Although the cartilage on the lateral femoral condyle was soft, it was not detached from subchondral bone. It could be considered as grade I according to the Outerbridge classification and the patient experienced no symptoms on the lateral side. It was thus decided to treat the lesion on the lateral condyle conservatively. The patient was kept non weight-bearing for six weeks and range of motion was started the day after surgery.

\section{Discussion}

OCD is the most common cartilage lesion in the knee and more than $70 \%$ of OCD is found on the posterolateral portion of the medial femoral condyle. Only in 15\%-20\% of cases has OCD been found on the central-inferior portion of the lateral femoral condyle $^{1)}$, and adult bilateral OCD of the femoral condyles is extremely rare. Cahill ${ }^{2}$ reported that bilateral involvement in patients with OCD of the knee has been reported in up to $25 \%$ of cases, but the lesions were often asymmetric in terms of size, location and symptomatology. However, no cases of bilateral OCD affecting femoral condyles in both knees have been described before, especially in two siblings.

OCD can be observed in any joint, but the exact etiology remains unclear. Traumatic, genetic and ischemic causes have all been described as possible etiologies. Ribbing ${ }^{3)}$ suggested that OCD represents separation of an accessory ossific nucleus of the distal femoral epiphysis, which partially attaches during maturation, but may completely separate if exposed to trauma. Our patients exhibited bilateral condylar involvement of both knees, but symptoms developed on one side in each patient. This unusual pattern could indicate that defects in the ossification centres of the distal femur, rather than ischemia or trauma, may have been the cause in these cases.

The genetic theory is also supported, as a high percentage 
members of the same family are found to have OCD of the $\mathrm{knee}^{4)}$. Genetic factors are considered to affect the structural integrity and pathophysiology ${ }^{5}$. Several identical twin studies demonstrated the influence of genetic factors on OCD pathogenesis and suggested that an underlying genetic predisposition may play a role ${ }^{5,6}$. We found OCD affecting bilateral femoral condyles of both knees in two siblings. Although we could not confirm the mode of inheritance because the remainders of the family were asymptomatic and refused clinical and radiographic examination, we consider that genetic factors remain essential as one of the main causes.

Treatment of OCD of the knee is a challenge for both physicians and patients. Numerous procedures exist to treat OCD, but which treatment constitutes the gold standard remains a topic of debate. Wright at al. ${ }^{7)}$ reported on 17 patients with OCD and showed that excision of OCD fragments resulted in a high percentage of fair and poor outcomes. Accordingly, they recommended against excision of loose fragments in an effort to preserve articular cartilage.

While juvenile OCD can usually be treated conservatively ${ }^{8,9)}$, surgery is generally required in adult cases. Retention of fragments is a goal for OCD treatment. If preservation of the fragment is not possible, there is an agreement that the large cartilage defect should be filled with osteochondral autograft or allograft plugs.

Various methods have been used for internal fixation ${ }^{9-12)}$ of displaced fragments in OCD. In this report, we have shown that a loose fragment secondary to OCD of the knee can be compressed using two Acutrak screws. Additionally, healing can be encouraged in a manner similar to that gained with the use of a Herbert screw in scaphoid fractures. In our male patient, it was possible to fix the fragment back to its femoral origin because the base was noted to be fresh. However, it was difficult to determine whether to prophylactically fix the cartilage on the lateral side or wait until the area of asymptomatic cartilage separated from the condyle to intervene. We chose to pursue conservative treatment in this case with the lack of clinical symptoms on the lateral side. In our female patient, internal fixation was deemed impossible due to the degenerative nature of the crater base and the lower likelihood of fragment healing even with the use of compression screws. A decision was made to proceed with ACI for lateral femoral lesion and OAT for medial lesion. Our clinical outcome shows that attempts to restore the integrity of the articular cartilage with a combination of osteochondral autografts and ACI for bicondykar OCD lesions in the same knee produce favorable results.
Surgical treatment of OCD of the knee is considered after establishing that the patient's symptoms are consistent with a full-thickness cartilage defect and after adequate nonoperative management has failed to provide acceptable pain relief ${ }^{13}$. In our two patients, the opposite sides were asymptomatic despite radiographic evidence of lesions. We decided not to treat the asymptomatic side, but both patients will be followed up on a regular basis for close monitoring of the onset of pain in the asymptomatic knee.

\section{Conflict of Interest}

No potential conflict of interest relevant to this article was reported.

\section{References}

1. Kocher MS, Tucker R, Ganley TJ, Flynn JM. Management of osteochondritis dissecans of the knee: current concepts review. Am J Sports Med. 2006;34:1181-91.

2. Cahill BR. Osteochondritis dissecans of the knee: treatment of juvenile and adult forms. J Am Acad Orthop Surg. 1995;3: 237-47.

3. Ribbing S. The hereditary multiple epiphyseal disturbance and its consequences for the aetiogenesis of local malacias: particularly the osteochondrosis dissecans. Acta Orthop Scand. 1955;24:286-99.

4. Stougaard J. Familial occurrence of osteochondritis dissecans. J Bone Joint Surg Br. 1964;46:542-3.

5. Onoda S, Sugita T, Aizawa T, Ohnuma M, Takahashi A. Osteochondritis dissecans of the knee in identical twins: a report of two cases. J Orthop Surg (Hong Kong). 2012;20: 108-10.

6. Mei-Dan O, Mann G, Steinbacher G, Cugat RB, Alvarez $\mathrm{PD}$. Bilateral osteochondritis dissecans of the knees in monozygotic twins: the genetic factor and review of the etiology. Am J Orthop (Belle Mead NJ). 2009;38:E152-5.

7. Wright RW, McLean M, Matava MJ, Shively RA. Osteochondritis dissecans of the knee: long-term results of excision of the fragment. Clin Orthop Relat Res. 2004;(424):239-43.

8. Cahill BR, Phillips MR, Navarro R. The results of conservative management of juvenile osteochondritis dissecans using joint scintigraphy: a prospective study. Am J Sports Med. 1989;17:601-5.

9. Dervin GF, Keene GC, Chissell HR. Biodegradable rods in adult osteochondritis dissecans of the knee. Clin Orthop 
Relat Res. 1998;(356):213-21.

10. Johnson LL, Uitvlugt G, Austin MD, Detrisac DA, Johnson C. Osteochondritis dissecans of the knee: arthroscopic compression screw fixation. Arthroscopy.1990;6:179-89.

11. Lindholm S, Pylkkänen P, Osterman K. Fixation of osteochondral fragments in the knee joint: a clinical survey. Clin Orthop Relat Res. 1977;(126):256-60.
12. Marcacci M, Kon E, Zaffagnini S, Visani A. Use of autologous grafts for reconstruction of osteochondral defects of the knee. Orthopedics. 1999;22:595-600.

13. Gomoll AH, Farr J, Gillogly SD, Kercher J, Minas T. Surgical management of articular cartilage defects of the knee. J Bone Joint Surg Am. 2010;92:2470-90. 